\title{
Particularities of fully-parallel manipulators in 6-DOFs robots design: a review of critical aspects
}

\author{
Lucian Milica ${ }^{1, *}$, and Gabriel Andrei ${ }^{1}$ \\ 1"Dunărea de Jos" University, Faculty of Engineering, 800201, Galati, Romania
}

\begin{abstract}
A whole range of industrial applications requires the presence of parallel mechanisms with six degrees of freedom (6-DOF) which have been developed in the last fifteen years, and one of the reasons why they still are a current topic is that present-day computers are capable of performing real-time motion laws of great complexity associated with these types of parallel mechanisms. The present work underlines particularities of parallel manipulators and their importance in the design of 6-DOF robots. The paper reveals the progress made in the last twenty years in the development of 6-DOF parallel manipulators, which increasingly find a wide scope of applications in different industrial areas such as robotics, manufacture and assisted medicine. It also emphasizes the need to determine singular configurations and the effect of cinematic redundancy which can increase the working space of the manipulators by adding active joints in one or more branches of the manipulator. Throughout the work, there were outlined three types of singularities encountered in the modelling of different types of parallel manipulators, and three types of redundancy. Furthermore, an analysis was made of the dimension of the workspace for a series of parallel manipulators, highlighting a number of factors that influence its size.
\end{abstract}

\section{Introduction}

In industry, the need to perform manifold operations under accurate handling and indefinite repetition has led to the emergence of automated devices with numerical control, which took over the monotonous work of human operators. The increase in flexibility of industrial processes through frequent and fast modification of the operating plan carried out by these devices has required their rescheduling. Thus, the first industrial robots appeared in the very sense of the term. With the emergence and development of computing, the numerical control of these devices was achieved by easier to-use and low-cost computer means.

It is well known that parallel manipulators are much stiffer and more accurate than series manipulators [1]. Thus, in recent years, in more technical applications parallel manipulators have been introduced, such as flight simulators, radar antennas, telescopes, pointing devices [2]. In addition, parallel manipulators have also been introduced in the

\footnotetext{
* Corresponding author: milica.lucian@ugal.ro
} 
processing centers with multiple degrees of freedom and high speed. The development of parallel manipulators with 6-DOFs took a greater momentum especially in the last fifteen years, starting as a basis for the research work carried out by a number of researchers in the development of parallel mechanisms with three degrees of freedom. Some researchers [3, 4] identified as such three types of planar parallel manipulators (Fig.1), consisting of three kinematic chains which link the mobile platform with the fixed one. These manipulators are distinguished among themselves through different types of kinematic joints which make the three kinematic chains mentioned above. Thus, it can be distinguished parallel manipulators type 3-RPR (revolute joint-prismatic joint-revolute joint), 3-RRR (three revolute joints) and 3-PRR (a prismatic joint and two revolute joints).
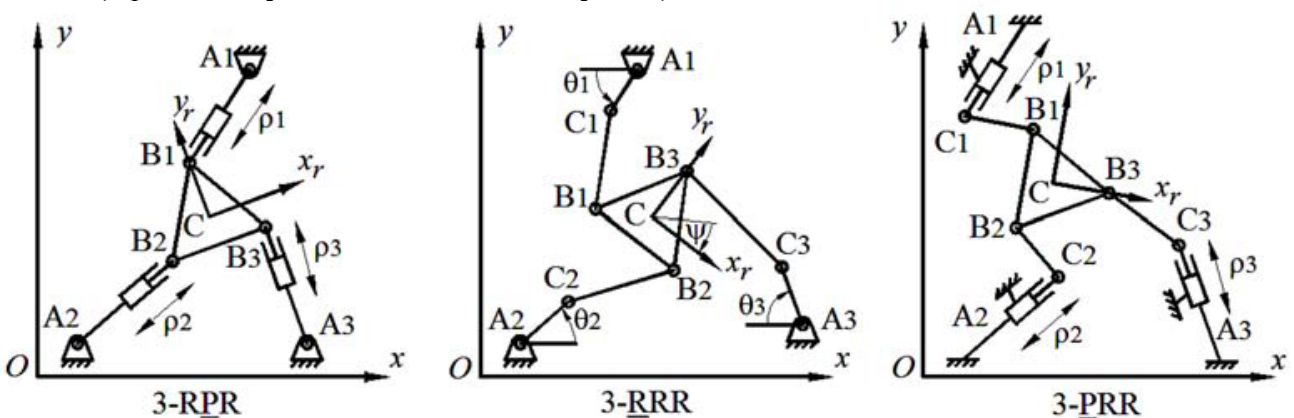

Fig. 1. Different types of planar parallel manipulators $[3,4]$

Jaime Gallardo et al. [5] studied spherical parallel manipulators, making reference to the families of spherical parallel manipulators with two arms. The main characteristic of this family is a compact asymmetric topology consisting of two arms and a spherical joint. The topology of the spherical manipulators is best expressed through the definition, advanced by the International Federation for Theory of Machines and Mechanisms (IFToMM), of this type of parallel manipulator: "manipulator parallel, which controls movement of the end effector via at least two linkages, starting from the end effector to the base ". Jaime Gallardo et al. described a transformation of a Stewart platform, into a spherical parallel manipulator (SPM), with a simplified structure. The position of the mobile platform is controlled by three arms with a common spherical joint attached to the mobile platform, while its orientation is controlled by the remaining arms.

A spherical parallel manipulator is a parallel manipulator whose mobile platform is an element with fixed point. Therefore all points attached to the mobile platform are moving on concentric spheres (Di Gregorio [6]). Several SPM with different architectures have been analyzed in the literature. However, due to two factors, to simplify the manufacturing process and the belief that a symmetric architecture is absolutely necessary for the proper performance of a manipulator, most of these contributions propose parallel manipulators with identical arms. On the other hand, since the robotic systems must be both flexible and capable of operating under different conditions, the natural question arises whether that "symmetry" is indeed essential in the design process. A typical SPM is a so-called "agile eye" which has followed the pioneering work of researchers Gosselin and Angeles [7] and contributed significantly to the further development of a SPM. In their work, Zhang et al. [8], using spherical passive joints along with four connectors, were able to determine, due to redundancy, the only possible orientation of the moving platform. In his work, Wohlhart [9], analyses the movement of a Gough-Stewart platform, where the displacement of the mobile platform is defined by a translation and a rotation movement.

In order to generate a class of spherical joints, Innocenti and Parenti-Castelli [10] have removed three translation joints, preserving the spherical passive joint, thus connecting the mobile platform with the fixed platform. However, such an architecture requires a strategic 
positioning of the passive spherical joint. For example, if the center of the spherical passive joint is placed in the geometric center of the triangle formed by spherical joints remaining attached to the mobile platform, the mobility mechanism can be badly affected and it can be easily transformed into a structure. Parallel manipulators type RPS (revolute joint-prismatic joint-spherical joint) represent another class of parallel manipulators, which have been dealt with by a number of researchers in different aspects related to the analysis of dynamic and kinematic behavior [11-13]. A particular approach to kinematic and dynamic problems of the manipulators type RPS is that of using the screw theory which proved to be an effective method for determining kinematic characteristics, including instant movements of the mechanism [14-19]. Also, one should not miss the fact that the parallel manipulators present some disadvantages such as: a limited workspace, different kinematic characteristics for each axis of the joints, the presence of singularities inside the workspace. For these reasons, many researchers sought to explore new approaches that would lead to an increase of the workspace, maximizing mechanism isotropy and avoiding internal singularities [20-23].

\section{Advantages of using parallel mechanisms}

Many applications in precision engineering (lithography, electron beam microscopy, microscopic assembly, aerospace industry, medical applications) require high-precision positioning in order to manipulate an object in different environments (vacuum or moisture). In the medical field, precision surgical instruments are required to avoid tissue damage during operation [24]. All the applications aforementioned refer to different environments, with certain characteristics. That is why, for example in wet environments, it is difficult to use conventional bearings for lubrication reasons. To overcome these problems, a number of mechanisms have been developed to satisfy both the requirements of accuracy and reliability. In most of the mentioned areas, it is necessary to manipulate an object within a space with six dimensions, which implies that the manipulator should have six degrees of freedom.

In general, this type of parallel robots is made up of two significant parts connected to each other through several elements. One is arbitrarily designated as fixed and is called the base plate, while the other one is connected via multiple kinematic chains at the base and is called motion platform or mobile platform. Robot elements are connected to each other through the spherical, cylindrical, revolute or prismatic joints. Usually, a parallel mechanism is symmetrical if it meets the following conditions: the number of branches is equal to the number of degrees of freedom corresponding to the mobile platform. An actuating device controls every branch, and the location and number of active joints of the arms are the same [25].

Parallel robots are generally defined as closed kinematic chain mechanisms comprising a terminal body with $n$ degrees of freedom (mobile platform) linked to a fixed platform through $n$ independent kinematic chains. According to some authors, some restrictions are necessary: each kinematic chain consists of maximum two articulated segments; the operation is performed by $n$ simple driving elements, one for each kinematic chain excluding redundant parallel manipulators where the number of actuators is higher than the spatiality degree of the mobile platform movement. For over two decades, parallel robots have attracted the attention of many researchers who consider them as being particularly valuable in terms of construction alternatives for robotic mechanisms [26, 27]. Various types of architectures of these mechanisms [28] have recently been studied and many are regularly used in the industrial world, as is the case of various types of machine tools [29] and industrial robots [30]. Parallel robots can be encountered especially in technical applications where a high speed motion of a rigid body in space is desired. Positioning and 
precision in executing the tasks are essential, because these robots are generally intended for handling of fragile objects; any error in the positioning of the tool could result in costly damages.

Parallel robots can be driven through actuators based on electric, hydraulic or pneumatic effect. Regardless of the type of actuation - electric, pneumatic or hydraulic - the presence of transducers is compulsory because it is absolutely essential to know accurately the relative position of the kinematic chain elements. The reason why parallel mechanisms are of great topicality is that present-day computers are able to solve complex calculations in real-time as required by the algorithms related to the parallel mechanisms.

Parallel mechanisms have a robust construction and can move bodies of considerable mass and size at high speeds, establishing a translational motion or rotation of the mobile platform. Compared to serial robots, parallel robots have some special features:

- high rigidity;

- running;

- high dynamic loading capacity;

- chain closed structure ensuring the acquisition of passive and active tasks much more efficiently;

- high positional accuracy.

Thanks to these features, parallel manipulators have been introduced within the machining centers with numerical control. It has been demonstrated by some researchers that a full analysis of the performance of parallel manipulators requires the determination of static and dynamic stiffness. In this direction, by analyzing the performance of a tripod-type parallel manipulator, Zhou and $\mathrm{Xi}$ [31] have carried out a finite element analysis to determine the vibration occurring in a technological process while Hong et al. [32] have investigated the stability of a machine tool whose table is positioned through a parallel manipulator.

\section{Kinematic and dynamic analysis}

The class of robots known as the Stewart platform has been implemented in the development of flight simulators [33-35]. Parallel Delta robot prototype [36, 37], the Tsai mechanism [38], as well as parallel robot star-type [39], are equipped with three actuators to move the mobile platform, giving it its three degrees of freedom.

Mobility is the main parameter of a structural mechanism and also one of the fundamental concepts in modeling dynamic and cinematic mechanisms [40]. IFToMM defines mobility as "the necessary number of independent coordinate system to define the configuration of a kinematic chain or mechanism" [41]. Mobility is, in fact, that which verifies the existence of a mechanism to impose the condition $M>0$.

In calculating the mobility mechanism the following parameters are generally used:

- number of joints $p$;

- number of kinematic elements $n,(n=m-1)$;

- total number of elements $m$, including fixed base;

- mobility $M$;

- degrees of freedom of kinematic joints $f_{i}$.

Gogu has advanced a new formula for the calculation of mobility parallel mechanisms, with the number of arms $t \geq 2$ [42].

$$
M=\sum_{i=1}^{p} f_{i}-\sum_{i=1}^{t} S_{i}+S_{p}
$$

where $f_{i}$ represents degrees of mobility of kinematic pairs $i(i=1, \ldots, p), S_{j}$ represents the degree of spatiality of $j$ arms considered isolated from the rest of the mechanism, and $S_{p}$ represents the degree of spatiality of the mobile platform. The degree of spatiality of the 
mobile platform depends on its links with the fixed base and it is given by the maximum number of independent motion parameters between the two. Mobility indicates the number of independent parameters both from the kinematic model and dynamic model; on the basis of these parameters it may be determined the number of inputs required by the actuation mechanism.

Gosselin and Angeles [43] have developed kinematic and dynamic straight model Agile Wrist, which is characterized by three concurrent rotations.

Kinematic analysis is carried out in two ways: via direct kinematic method and by inverse kinematic method. The transformation by which one can determine the instantaneous movement of mobile platform, as a function of relative velocities of the actuated joints, represents direct kinematic method. The transformation has the expression:

$$
\dot{\mathbf{q}} \rightarrow \dot{\mathbf{x}}
$$

where vector $\dot{\mathbf{q}}=\left[q_{1} \ldots q_{m}\right]^{T}$ is the vector of generalized coordinates (parameters of its components being $q_{j}$ joints), and the vector $\mathbf{x}=\left[\begin{array}{lllll}\alpha \beta & \gamma & x & y & z\end{array}\right]^{T}$ describes the position of the mobile platform, where $x, y, z$ are the coordinates of the characteristic point in relation to the fixed reference system, and $\alpha, \beta, \gamma$ are the angles of orientation in relation to the same fixed reference system.

Inverse kinematic method consists in determining the movements of the actuated joints, knowing the movement imposed on the mobile platform. The transformation in this case has the expression:

$$
\dot{\mathbf{x}} \rightarrow \dot{\mathbf{q}}
$$

Dynamic analysis of parallel robots is usually approached by the analytical method of classical mechanics. Dasgupta and Mruthyunjaya [44] used Newton-Euler method to develop dynamic equations of Stewart platform, taking into account both the gravity and dynamic effect and friction that occur in joints.

Tsai [45] has also presented an algorithm to solve the inverse dynamics of a Stewart platform, using the same Newton-Euler method.

It is known that this approach leads to a set of equations, but they are very difficult to use in order to obtain the laws of advanced control. At the same time, quite few results were obtained by specific dynamic modeling of the parallel mechanisms.

Kane and Levinson [46] have obtained some recursive relations with respect to the balance of the generalized forces which apply to an arm of the serial robot. Kane's dynamic equations are described by means of a Stanford robot. Sorli et al. [47] have also provided a dynamic modeling for a Turin parallel robot, which has three identical legs and six degrees of freedom.

In some simplifying assumptions on the geometry and distribution of robot inertia, Geng et al. [48] and Tsai and Stamper [49] have developed a system of Lagrange equations of motion.

Determining the mechanical behavior of the manipulator according to the mechanism configuration calls for an optimal design of the operation and control system. Lagrange equations are systems of second-degree differential equations that determine the dynamics of parallel manipulator. Starting from the basic equations:

$$
\frac{d}{d t}\left(\frac{\partial L}{\partial \dot{\mathbf{x}}}\right)-\frac{\partial L}{\partial \mathbf{x}}+\frac{\partial \Phi^{T}}{\partial \mathbf{x}} \lambda=\mathbf{f}
$$

Nguyen, Bouzgarrou et al. [50] determined all structural parameters of the robot (mutual kinematic constraints, size of components). Also, the results thus obtained were compared with data provided by ANSYS finite element analysis software.

Analytical calculation involving Lagrange method is still too long and the risk of errors is high [51]. It turns out that the time for numerical calculation increases with the number of elements. Therefore, this calculation method is impossible to be done in real-time, being practically unusable. 


\section{Singular configurations}

In fact, most existing parallel robots have limited workspace and its calculation is quite complicated because of singularities (those configurations in which instantaneous complete movement of mechanism cannot be determined). Many of the recent works focuses on avoiding critical positions that can handle the mechanism where the workspace is reduced and the problem of finding an algorithm to determine a route that avoids singular configurations becomes complex [52-54].

Whenever is wanted to design a parallel manipulator, it is very important to understand the intrinsic nature of singularities and their relationships with the robot kinematics and configurations [55]. Currently, there are many different points of view on the singularity phenomena encountered in the kinematics of the parallel mechanisms. The first study on the positions of singularity encountered in parallel mechanisms date back to 1970 when Hunt [56], in his study, defined two types of singular configurations: stationary configurations and uncertain configurations. Merlet [57] proposed another approach for the determination of singular configurations through the geometry of Grassmann, one of the founders of the vector geometry and the multidimensional geometry, who assumed the existence of a space of $\mathrm{n}$ dimensions. Later, Gosselin and Angeles [58] defined three types of singularities in terms of the rank of matrix. These authors brought important contributions on this issue. In general, singular configurations for parallel manipulators are achieved when the determinants of different Jacobian matrixes are zero. It has been illustrated and classified different types of singular configurations. Thus, two types of outstanding singularities were highlighted: the inverse kinematical singularity and direct kinematical singularity which are used to study and analyze the parallel manipulators [59]. Another approach to the problem of singular positions was highlighted by Zlatanov, Bonev et al. [60], using the screw theory while Wolf and Shoham [61] have investigated these issues through the linear complex approximation.

Singularities are important aspects to be considered when talking about parallel robots. To determine these critical positions is to identify them and avoid them by ensuring the kinematic stability and accuracy of the system. Many robot manipulators have six degrees of freedom and are able in nonsingular positions to generate a space with six dimensions for an infinitesimal motion to end effector /mobile platform [62]. In a singular position the axes of the joints generate a movement space for five of the six axes so that even an infinitesimal motion of translation or rotation is not possible. This single position can be determined by placing the condition that the determinant of the Jacobian matrix $(6 \times 6)$ be equal to 0 . Another way to describe singularities is through geometric line providing a qualitative approach to them $[63,64]$. Singular positions correspond to the positions in which the Plücker coordinates robot of the axes of the joints form a dependent linear system, and these situations can be characterized in a pure geometric way [65]. In this context, a prismatic joints axis, for example, is defined as an "infinite line". The algorithm used for instance, by Stoica et al. [66] for the analysis of singular configurations is based on the determinants of the two Jacobian matrixes, $\mathbf{A}$ and $\mathbf{B}$, derived from the geometric model and the inverse geometric model. Using the matrix representation, there is the following expression:

where:

$$
\frac{\partial \mathrm{f}}{\partial \mathbf{x}} \dot{\mathbf{x}}-\frac{\partial \mathrm{f}}{\partial \mathbf{q}} \dot{\mathbf{q}}=0 \Rightarrow \mathbf{A} \cdot \dot{\mathbf{x}}+\mathbf{B} \cdot \dot{\mathbf{q}}=0
$$

- $\mathbf{q}$ is generalized coordinates vector with its components forming the robot space;

- $\mathbf{x}$ is the position vector of a point of the mobile platform and angles $\phi, \theta$ and $\psi$ give its orientation; 
- $\mathbf{f}$ is an $n$-dimensional vector, including geometric constraints of each kinematic chain $i$, for $i=1,2 \ldots, n$.

Thus, some authors have identified three types of singularities [67, 68]:

- singularity of type I, is the first type of singularity which occurs when the determinant of the Jacobian matrix $\mathbf{B}$ is zero. If the matrix determinant $\mathbf{B}$ is zero, the manipulator loses one or more degrees of freedom ;

- a second type of singularity (singularity of type II) occurs when the determinant of the Jacobian matrix A equals 0. In this case the robot wins one or more degrees of freedom.

- singularity of type III occurs when determinants require both matrixes to be zero. These types of singularities are encountered in the literature as the architectural singularities.

In these particular positions, the degree of spatiality of the effector motion decreases, and therefore some of the input parameters have no effect on its movement. The problem of critical position is the subject of special studies on the manipulator mechanisms. Thus BenHorin and Shoham [69] performed an analysis of singularities, for a series of parallel robots through Grassmann-Cayley algebra. Tong Z. et al. [70] highlight the appearance of singularities for a category of symmetric Gough-Stewart manipulators.

These studies determine the set of critical positions which sometimes form "surfaces" on the workspace, thereby delimiting the size of this space.

\section{Redundancy of parallel mechanisms}

A new development direction for 6-DOFs parallel manipulators is the micro-manipulators. Since 1989, Hara [71] proposed a 6-SPS micro-manipulator parallel which uses a piezoelectric ceramic actuator. In 1990, Taniguchi [72] developed a new parallel micromanipulator type 6-PSS. In his research Yun and Li [73] refer to the features of a 6-DOFs parallel manipulator with piezoelectric actuators and dual redundancy (Fig. 2).

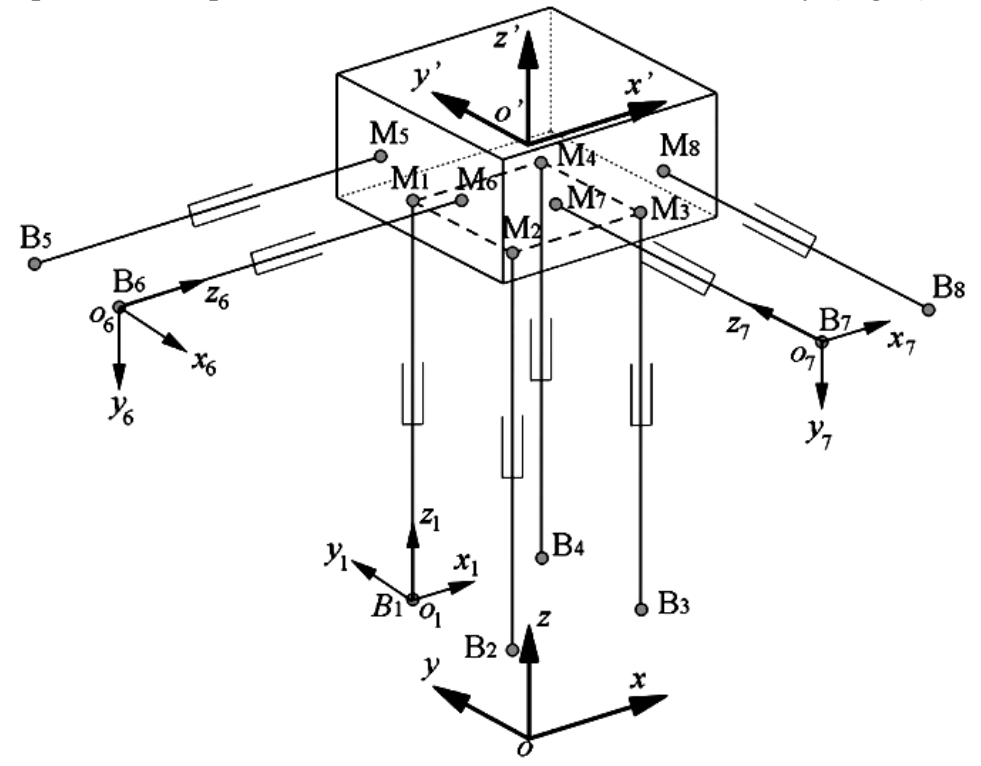

Fig. 2. Parallel manipulator with dual redundancy, type 8-PSS [73]

The work of Yun and Li offers suggestions for improving the structure of the parallel redundant mechanism and gives solutions for the optimization of a control algorithm, in order to achieve a possible workspace, more accurate motion and dynamically superior 
features. The existence of redundant movements enables the execution of a desired movement in several ways, which creates the possibility of optimizing the operation of the robot. The degree of redundancy is the consequence of the particular relative positions of axes couplings [74]. Moreover, according to recent research, the redundancy is a way to avoid singular configurations [75].

The redundancy of parallel manipulators can be of three types: actuators redundancy, arms redundancy and kinematic redundancy $[76,77]$. Actuators redundancy consist in replacing passive joints with active joints $[78,80]$ in which case both the number of degrees of freedom and the manipulator's mobility remain unchanged. The redundancy of arms can improve the strength capabilities of the manipulator and can reduce the number of singular configurations. The kinematic redundancy can increase manipulator workspace by adding one or more active joints to the manipulator arms [81, 82]. In addition it allows kinematic redundancy manipulator to follow trajectories that are far from singular configurations.

The degree of redundancy depends clearly on the degree of mobility and the degree of spatiality of the analyzed mechanism; it represents the number of mobility remaining to the mechanism after immobilizing the effector element. The existence of redundant movements enables the execution of a desired movement in several ways, which further creates the possibility to optimize the operation of the robot.

Another example illustrating the influence of the degree of redundancy on parallel mechanisms with six degrees of freedom is the manipulator type 6(P) UPS which is a mechanism derived from Stewart-Gough mechanism, [83] and manipulator type 6(P) RUS $[84,85]$.

Position and orientation of the mobile platform are given by the $\mathbf{x}$ vector that has the expression:

$$
\mathbf{x}=\left[\begin{array}{llllll}
x_{\mathbf{E}} & y_{\mathbf{E}} & z_{\mathbf{E}} & \alpha_{\mathbf{E}} & \beta_{\mathbf{E}} & \gamma_{\mathbf{E}}
\end{array}\right]^{T}
$$

where $\mathbf{x}_{\mathrm{E}}, \mathbf{y}_{\mathrm{E}}$ and $\mathbf{z}_{\mathrm{E}}$ represent projections of the position vector $\mathbf{r}_{\mathrm{E}}$ belonging to the $(\mathrm{CF})_{\mathrm{E}}$ system on the $(\mathrm{CF})_{\mathrm{O}}$ system and $\alpha_{E}, \beta_{E}, \gamma_{E}$ give the orientation of $(\mathrm{CF})_{\mathrm{E}}$ system around the $x, y, z$ axes of the $(\mathrm{CF})_{\mathrm{O}}$ system. $(\mathrm{CF})_{\mathrm{E}}$ and $(\mathrm{CF})_{\mathrm{O}}$, are the notations used for the reference system of the mobile platform respectively for the reference system of the frame. The input data are entered by the vector $\mathbf{q}_{1}$ for the manipulator type 6(P) UPS [83] and $\mathbf{q}_{2}$ vector for the manipulator type 6(P) RUS [84, 85]:

$$
\mathbf{q}_{\mathbf{1}}=\left[\rho_{1} \rho_{2} \rho_{3} \rho_{4} \rho_{5} \rho_{6} \delta\right]^{T}
$$

and $\rho_{j}$ is the actuating $i$ elements length,

$$
\mathbf{q}_{2}=\left[\theta_{1} \theta_{2} \theta_{3} \theta_{4} \theta_{5} \theta_{6} \delta\right]^{T}
$$

where $\theta_{j}$ represents the angular displacement of $l_{i}$ arms.

In case of both manipulators, the position of $G_{1}$ is given by the reduntant actuator's $\delta$ displacement ( $G_{1}$ is a point that belongs to the redundant joint).

\section{Workspace volume}

For the evaluation of a parallel manipulator performance, workspace represents an important criterion. Typically, the degree of difficulty for setting workspace grows proportionally with the increase in the number of degrees of freedom of the parallel manipulator. In the present review, the workspace volume has been considered and also, a comparative study for a series of parallel manipulators with six degrees of freedom was done. Fully working space for parallel manipulators with six degrees of freedom is a space with six dimensions, whose representation is difficult to express for human perception [86]. Therefore there are typically determined the subspaces of this space. These subspaces are three-dimensional spaces that can be defined within the spatial Cartesian coordinate system. The workspace of the robot can be briefly defined as the volume generated by a point of the 
effector element when the mechanism takes all possible configurations. It can be classified into two categories: reachable workspace and orientation workspace [87].

The reachable workspace is determined by the points reached by the end effector when the actuating branches go through all the possible combinations of their rotation domain. The orientation workspace is determined by the possible orientations of the end effector. It is known that an orientation of any system $\left\{R_{1}\right\}$, relative to the other system $\left\{R_{2}\right\}$ is expressed using Euler angles. The final equation of the rotation matrix related to Euler angles is obtained by three successive elementary rotations applied to $\left\{R_{1}\right\}$ system, by angles $\phi, \theta$ and respectively $\psi[88]$. Euler angles allow the representation of the orientation workspace for most parallel manipulators as volumes with simple form. Using cylindrical coordinate representation for the orientation workspace, Merlet [89], obtains a graph of the volume of this space as a function of angle variation $\psi$ (Fig.3), highlighting the fact that for different values assigned to the angles $\phi$ and $\theta$ orientation workspace volume increases as the angle value $\psi$ approaching $0^{\circ}$.

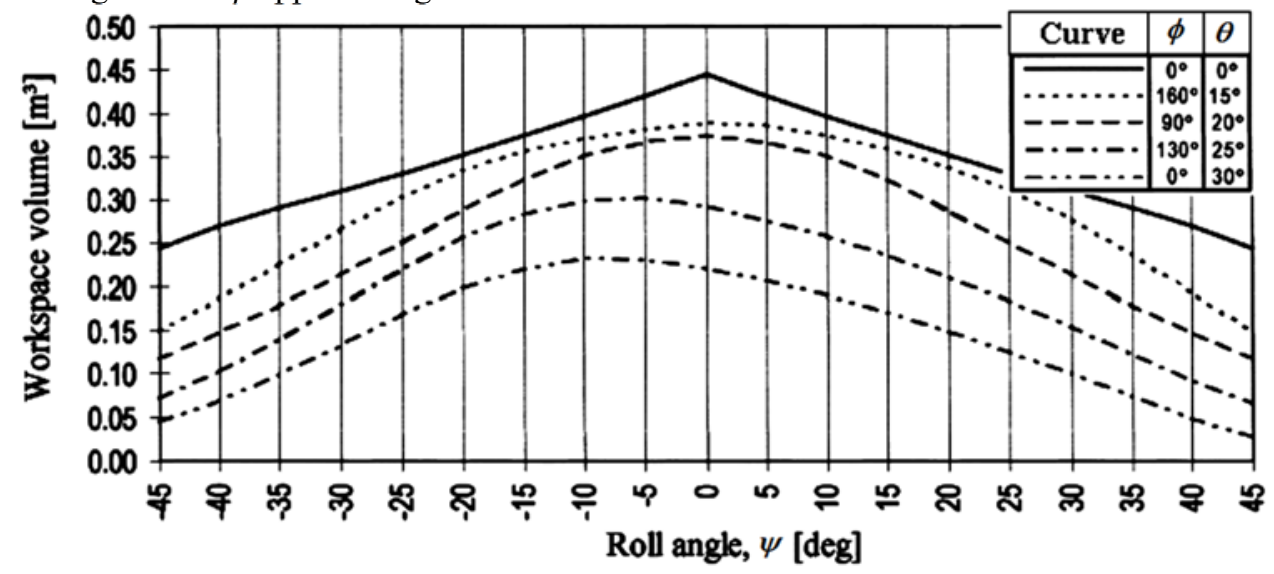

Fig. 3. The volume of the orientation workspace depending on the angle $\psi$ [89]

Bonev and Ryu [90] propose a computational algorithm, implemented with MATLAB software application, in order to determine the influence of the characteristic position of point $C$ (belonging to mobile platform), in relation to the axis of symmetry of a parallel manipulator, over the orientation workspace described. Yi Lu et al. [91] analyzed a new kinematic model of Stewart type manipulator, determining the influence of angular rotation of spherical joints. They highlight the following differences with respect to the workspace volume, compared to Stewart platform (Tab.1):

Table 1. Workspace volume, compared to Stewart platform [91].

\begin{tabular}{|c|c|c|c|c|c|}
\hline P.M. type & $\begin{array}{c}e \\
{[\mathrm{~mm}]}\end{array}$ & $\begin{array}{c}E \\
{[\mathrm{~mm}]}\end{array}$ & $\begin{array}{c}\theta \\
{[\mathrm{deg}]}\end{array}$ & $\begin{array}{c}r_{i j} \\
{[\mathrm{~mm}]}\end{array}$ & $\begin{array}{c}V_{w} \\
{\left[\mathrm{~mm}^{3}\right]}\end{array}$ \\
\hline Yi Lu & 100 & 250 & - & $500-700$ & 71.905 \\
\hline Stewart & 100 & 250 & \pm 30 & $500-700$ & 20.793 \\
\hline Stewart & 100 & 250 & \pm 35 & $500-700$ & 33.585 \\
\hline Stewart & 100 & 250 & \pm 40 & $500-700$ & 48.745 \\
\hline Stewart & 100 & 250 & \pm 45 & $500-700$ & 58.947 \\
\hline Stewart & 100 & 250 & \pm 50 & $500-700$ & 77.163 \\
\hline
\end{tabular}

In the Table $1, e$ and $E$ are the distances from $B_{i}$ to $o$ and $B_{i}$ to $O$ respectively. $O$ and $o$ are the center point of $B, m$ ( $B$ represent the frame end $m$ represent mobile platform), $\theta$ is the limited rotational angle of the $S$ joint. In the case of Stewart manipulator, it can be 
observed, that with increased rotation angle of the spherical joint $\theta$ within $\pm(30 \ldots 50)^{\circ}$, the volume of the workspace increases as well. On the other hand, pairs of kinematic elements which make the link between mobile platform and fixed base plate have dimensions imposed by the mechanism configuration. Spherical joints that theoretically can achieve $360^{\circ}$ rotation around an axis, actually they are restricted in terms of the angular movement and for this reason it is necessary to determine the maximum value of the rotation angle $\theta$, for each kinematic joint, in the design stage [92].

Lim et al. [92] examine the workspace volume of a parallel manipulator with suspended mobile platform (Tab.2).

Table 2. Workspace volume of P.M. by Lim [93]

\begin{tabular}{|c|c|c|c|c|c|}
\hline P.M. type & $\begin{array}{c}L \\
{[\mathrm{~mm}]}\end{array}$ & $\begin{array}{c}\mu \\
{[\mathrm{deg}]}\end{array}$ & $\begin{array}{c}\gamma \\
{[\mathrm{deg}]}\end{array}$ & $\begin{array}{c}r \\
{[\mathrm{~mm}]}\end{array}$ & $\begin{array}{c}V_{w} \\
{[\mathrm{~mm} 3]}\end{array}$ \\
\hline $\operatorname{Lim}$ & 800 & 100 & 82 & 300 & 39.334 \\
\hline
\end{tabular}

Coppola G. et al. [94], in their study on the reconfigurable parameters of a parallel mechanism with six degrees of freedom, highlight its volume workspace (Tab.3):

Table 3. Workspace volume of P.M. by Coppola [94]

\begin{tabular}{|c|c|c|c|c|c|c|c|c|}
\hline $\begin{array}{c}\text { P.M. } \\
\text { type }\end{array}$ & $\begin{array}{c}b \\
{[\mathrm{~mm}]}\end{array}$ & $\begin{array}{c}r \\
{[\mathrm{~mm}]}\end{array}$ & $\begin{array}{c}\alpha \\
{[\mathrm{mm}]}\end{array}$ & $\begin{array}{c}l_{\min } \\
{[\mathrm{mm}]}\end{array}$ & $\begin{array}{c}l_{\max } \\
{[\mathrm{mm}]}\end{array}$ & $\begin{array}{c}\alpha_{\min } \\
{[\mathrm{deg}]}\end{array}$ & $\begin{array}{c}\alpha_{\max } \\
{[\mathrm{deg}]}\end{array}$ & $\begin{array}{c}V_{w} \\
{\left[\mathrm{~mm}^{3}\right]}\end{array}$ \\
\hline Coppola & 85 & 125 & 32,5 & 150 & 300 & 45 & 145 & 26.200 \\
\hline
\end{tabular}

In the Table $2, l_{\min }$ and $l_{\max }$ are minimum and maximum values of the actuating branch length, $a, b$, and $r$ are the geometrical values which correspond to the lengths of their respective vectors and $\alpha_{\min }$ and $\alpha_{\max }$ are the minimum and maximum values assigned to the angular movement of the joint.

It should also be pointed out that several works from the literature describe various ways to use CAD software to investigate the working space of parallel manipulators [9597]. On the basis of such methods, an approximate measurement of the inclination angle of the mobile platform could be made.

Another problem of parallel mechanisms, in general, is the motion limitation imposed on their kinematic elements in order to avoid any collision between them. Basically, these limitations determine a restricting workspace and the analysis of positions that may cause such collisions is a prerequisite for the proper functioning of the manipulator [98].

\section{Conclusions}

The present work reviews the main aspects of determining the workspace and avoiding singular configuration of 6-DOFs parallel manipulators, highlighting the importance of existing redundant movements within these types of manipulators. The paper also revealed the progress made in the last twenty years in the development of of 6-DOFs parallel manipulators which increasingly find a wide scope of applications in different industrial areas such as robotics and assisted medicine.

Throughout the work, there were outlined three types of singularities encountered in the modelling of different types of parallel manipulators, and three types of redundancy. Also, an analysis was made of the dimension of the workspace for a series of parallel manipulators, highlighting a number of factors that influence its dimensions:

- the design parameters $(r, l, a, b, e)$;

- the value of rotation angle $\theta$ of spherical joint, in the case of Stewart manipulators; 
- position of characteristic point $C$ (belonging to mobile platform), in relation to the symmetry axis of parallel manipulator;

- variation of the angles, $\phi, \theta$ and $\psi$;

- the motion limitation imposed on cinematic elements, to avoid collision between them.

Knowing the characteristics of parallel mechanisms with six degrees of freedom, a new direction for future research would be the design of a novel type of parallel manipulator, with six degrees of freedom, able to perform complex milling process; moreover, once designed such type of robot, the optimum parameters necessary to achieve a larger workspace have to be inferred.

\section{References}

1. J. P. Merlet, Parallel robots. 2nd ed., (2006)

2. D. Zhang, Parallel robotic machine tools, (2010)

3. J. P. Merlet, Tehnical Report 1645, INRIA, (1992)

4. C. Gosselin, J. Angeles, J. Mech. Transm.-T. ASME, 110, 35-41 (1988).

5. J. Gallardo, R. Rodrıguez, M. Caudillo, J. M. Rico, Mech. Mach. Theory, 43, 201-216 (2008)

6. R. Di Gregorio, J. Mech. Transm.-T. ASME., 126, 850-855 (2004)

7. C. M. Gosselin, J. Angeles, J. Mech. Transm.-T ASME., 111, 202-207 (1989)

8. Y. Zhang, C. Crane, J. Duffy, J. Robotic Syst., 15, 299-308 (1998)

9. K. Wohlhart, Mech. Mach. Theory, 29, 581-589 (1994)

10. C. Innocenti, V. Parenti-Castelli, Mech. Mach. Theory, 28, 553-561 (1993)

11. K. M. Lee, D.K. Sha, Proceedings IEEE/ICRA., 1, 345-350 (1987)

12. H. S. Kim, L.W. Tsai, J. Mech. Transm.-T. ASME., 125, $92-97$ (2003)

13. C. H. Liu, S. Cheng, J. Mech. Des. ASME., 126,1006-1016 (2004)

14. Z. Huang, Y. Fang, Mech. Mach. Theory, 31, 1009-1018 (1996)

15. Y. Fang, Z. Huang, Mech. Mach. Theory, 32, 789-796 (1997)

16. Z. Huang, J. Wang, Mech. Mach. Theory, 36, 893-911 (2001)

17. S. K. Agrawal, Proceedings of 8th World Congress on TMM, 405-408 (1990)

18. Z. Huang, J. Wang, Proceedings of A Symposium Commemorating of Sir Robert Stawell Ball, (2000)

19. Z. Huang, J. Wang, Y. Fang, Mech. Mach. Theory, 37, 229-240 (2002)

20. J. Merlet, C. Gosselin, N. Mouly, Mech. Mach. Theory, 33, 7-20 (1998)

21. F. Bulca, J. Angeles, P.J. Zsombor-Murray, Mech. Mach. Theory, 34, 497-512 (1999)

22. A. Kosinska, M. Galicki, K. Kedzior, J. Robotic Syst., 20, 539-548 (2003)

23. M. Arsenault, R. Boudreau, J. Robotic Syst., 21, 259-274 (2004)

24. A. Dunning, G. Tolou, Int. J. Mech. Sc., 2, 157-168 (2011)

25. L.-W. Tsai, Robot Analysis:The Mechanics of Serial and Parallel Manipulator, (1999)

26. D. Chablat, P. Wenger, IEEE Trans. Robot. Autom., 19, 403-410 (2003)

27. X. J. Liu, J. Wang, F. Gao, L.P. Wang, IEEE Trans. Robot. Autom., 17, 959-968 (2001)

28. C. Reboulet, R. Pigeyre, Proceedings of the ISRAM., 293-298 (1990)

29. M. Valenti, ASME. Mech. Eng., 17, 70-75 (1995)

30. K. Cleary, T. Brooks, Proceedings of the IEEE/ICRA., 708-713 (1993)

31. Z. Zhou, J. Xi, J. Mech. Des. 128, 403-412 (2006)

32. D. Hong et al, Mech. Struct. Mach., 31, 509-528 (2003)

33. J. Angeles, Springer, (1997)

34. V. Parenti-Castelli, R. Di Gregorio,.J. Mech. Des., 122, 294-298 (2000)

35. D. Stewart. Proceedings of Int.Mech.Eng., 15, 371-386 (1965)

36. R. Clavel, Proceedings of the ISR., (1988) 
37. S. Staicu, D.C. Carp-Ciocardia, Proceedings of the IEEE/ICRA., 416-412 (2003)

38. L.W. Tsai, R. Stamper, ASME. Des. Eng. Tech. Conf. (1996)

39. J. M. Hervé, F. Sparacino, Proceedings of the ARK., (1992)

40. G. Gogu, Report ROBEA MAX CNRS, (2003)

41. T. G. Ionescu, Mech. Mach. Theory, 38 (2003)

42. G. Gogu, Mech. Mach. Theory, 40, 1068-1097 (2005)

43. C. Gosselin, J. Angeles, J. Mech. Transm.-T. ASME., 111, 202-207 (1989)

44. B. Dasgupta, T.S. Mruthyunjaya, Mech. Mach. Theory, 34, 1135-1152 (1998)

45. L.W. Tsai, Robot Analysis: The Mechanics of Serial and Parallel Manipulator, (1999)

46. T.R. Kane, D.A. Levinson, Dynamics, Theory and Applications, (1985)

47. M. Sorli, C. Ferarresi, M. Kolarski, B. Borovac, M. Vucobratovic, Mech. Mach. Theory, 32, 51-77 (1997)

48. Z. Geng, L.S. Haynes, J.D. Lee, R.L. Carroll, Robot. Autonom. Syst., 9, 237-254 (1992)

49. L.W. Tsai, R. Stamper, ASME. Des. Eng. Tech. Conf., (1996)

50. A.V. Nguyen, B.C. Bouzgarrou, K. Charlet, A. Béakou, Mech. Mach. Theory, 93, 65$82(2015)$

51. S. Staicu, X.J. Liu, J. Li, Springer, 58, 217-235 (2009)

52. B. Dasgupta, T.S. Mruthyunjaya, Mech. Mach. Theory, 33, 711-725 (1998)

53. C.L. Lin, H.Y. Jan, J.R. Lin, T.S. Hwang, Eur. J. Control, 3, 201-212 (2008)

54. S. Parsa, R. Boudreau, J.A. Carretero, Mech. Mach. Theory, 85, 53-63 (2015)

55. P.C. Lee, J.J. Lee, Mech. Eng., 7, 163-187 (2012)

56. K.H. Hunt, Cambridge University Press (1978)

57. J. P. Merlet, Int. J. Robot. Res., 8, 45-56 (1989)

58. C. Gosselin, J. Angeles, Proceedings of IEEE T. Robotic. Autom., 6, 281-290 (1990)

59. J. Craig, Introduction to Robotics, Mechanics and Control, Third Edition, (2005)

60. D. Zlatanov, I.A. Bonev, C. Gosselin, Proceedings of the IEEE/ICRA, 496-502 (2002)

61. A. Wolf, M. Shoham, J. Mech. Transm.-T. ASME., 125, 564-572 (2003)

62. R. Penne, E. Smet, P. Klosiewicz, J. Intell. Robot. Syst., 62, 205-216 (2011)

63. S. Staicu. Robotica, 27, 199-207 (2009)

64. F. Hao, J.M. McCarthy, J. Robot. Syst. 15, 43-55 (1998)

65. A. Dandurand, The rigidity of compound spatial grids., 10, 41-56 (1984)

66. A. Stoica, D. Pisla, S. Andras, B. Gherman, B.Z. Gyurka, N. Plitea, Mech. Eng., 8 ,7079 (2013)

67. C. Gosselin, J. Angeles, IEEE T. Robotic. Autom., 6, 281-290 (1990)

68. O. Ma, J. Angeles, Proceedings of the IEEE ICRA., 1542-1547 (1991)

69. P. Ben-Horin, M. Shoham, Mech. Mach. Theory, 41, 958-970 (2006)

70. Z. Tong, J.F He, H.Z. Jiang et al, Robotica, 30, 305-314 (2012)

71. A. Hara, K. Sugimoto, J. Mech. Transm.-T. ASME.,111, 34-39 (1989)

72. M. Taniguchi, M. Ikrda, A. Inagaki, Int. J. Jpn. Soc. Precis. Eng., 26, 35-40 (1992)

73. Y. Yun, Y. Li, Springer, 61, 829-845 (2010)

74. J. Wang, C. Gosselin, J. Mech. Transm.-T. ASME., 126, 109-118 (2004)

75. I. Ebrahimi, J.A. Carretero, R. Boudreau, Mech. Mach. Theory, 42, 1007-1016 (2007)

76. S. Lee, S. Kim, Proceedings of the IEEE/CDC., 2, 1097-1102 (1993)

77. R. Boudreau, S. Nokleby, Mech. Mach. Theory, 56, 138-155 (2012)

78. K.E. Zanganeh, J. Angeles, Proceedings of IEEE/ICRA., 3043-3048 (1994)

79. H. Cheng, G.F. Liu, Y.K. Yiu, Z.H. Xiong, Z. Li, Proceedings of the IEEE/IROS, 171$176(2011)$

80. J. P. Merlet, Redundant parallel manipulators, 8, 17-24 (1996)

81. J. Wang, C. Gosselin, J. Mech. Des., 126, 109-118 (2004)

82. J. Kotlarski, B. Heimann, T. Ortmaier, Mech. Eng., 7, 120-134 (2012) 
83. P. Last, C. Budde, J. Hesselbach,. Proceedings of the 2005 IEEE/CASE., 393-398 (2005)

84. Y. Jin, I.M. Chen, G.L. Yang, Mech. Mach. Theory, 44, 912-922 (2009)

85. E. Ottaviano, M. Ceccarelli, Optimal design of CAPAMAN with prescribed workspace, 35-44, (2001)

86. D.I. Kim, W.K. Chung, Y. Youm, IEEE/ICRA., 2986-2991 (1997)

87. I.A. Bonev, J. Ryu, Mech. Mach. Theory, 36, 15-28 (2001)

88. A. Năstase, Mecanica Roboţilor, 2, 24-30 (2012)

89. J.P. Merlet, Mech. Mach. Theory, 29, 1099-1113 (1994)

90. I.A. Bonev, J. Ryu, Mech. Mach. Theory, 36, 1-13 (2001)

91. Y. Lu, X. Li, C. Zhang, Y. Liu, Robotica, 1-16 (2014)

92. H. Lim, S.H. Lee, B.R. So, B.J. Yi, Int. J. Cont. Autom. and Syst., 13, 942-950 (2015)

93. M. Majid, Z.A. Huang, Y.L. Yao, Int. J. Adv. Manuf. Tech., 16, 441-449 (2000)

94. G. Coppola, D. Zhanga, K. Li, Robot. Cim.-Int. Manuf., 30, 99-106 (2014)

95. K. Arrouk, B. Bouzgarrou, G. Gogu, Appl. Mech. Mater., 162, 131-140 (2012)

96. K. Arrouk, B. Bouzgarrou, G. Gogu, RAAD., (2010)

97. K. Arrouk, B. Bouzgarrou, G. Gogu, Springer, 5, 605-612, (2010)

98. http://www.orbitmotionsystems.com., Operation Manual PacDrive Robot D2, Edition 2008-08 